\title{
Inflammation markers, neutrophil-to-lymphocyte ratio and platelet-to lymphocyte ratio after pulmonary vein isolation, were associated with late clinical outcomes and pulmonary vein reconnections
}

Masamichi Yano ${ }^{1}$, Yasuyuki Egami ${ }^{1}$, Kohei Ukita ${ }^{1}$, Akito Kawamura ${ }^{1}$, Hitoshi Nakamura ${ }^{1}$, Yutaka Matsuhiro $^{1}$, Koji Yasumoto ${ }^{1}$, Masaki Tsuda ${ }^{1}$, Naotaka Okamoto ${ }^{1}$, Akihiro Tanaka ${ }^{1}$, Yasuharu Matsunaga-Lee ${ }^{1}$, Ryu Shutta ${ }^{1}$, Masami Nishino ${ }^{1}$, and Jun Tanouchi ${ }^{1}$

${ }^{1}$ Osaka Rosai Hospital

April 28, 2020

\begin{abstract}
Background Previous reports demonstrated that the early recurrence of atrial fibrillation (ERAF) after pulmonary vein isolation (PVI) was associated with the inflammatory process. The neutrophil to lymphocyte ratio (NLR) and platelet to lymphocyte ratio (PLR) have been proposed as indicators of a systemic inflammatory response. However the relationship between NLR/PLR and clinical outcomes after PVI remains unclear. Methods and Results We enrolled 633 consecutive atrial fibrillation (AF) patients who underwent PVI from September 2014 to June 2018. The NLR and PLR in peripheral blood leukocytes 1 day before and 36-48 hours after PVI were measured. We compared the NLR and PLR changes in each parameter $(\Delta N L R$ and $\Delta$ PLR) between a late recurrence of AF group after the PVI (LRAF, during 1 year after PVI) and the no-LRAF group. We also evaluated the relationship between the $\triangle \mathrm{NLR} / \triangle \mathrm{PLR}$ and pulmonary vein (PV) reconnections in repeated ablation procedures. The LRAF patients consisted of 151 patients $(23.9 \%)$. The $\triangle$ NLR and $\triangle$ PLR were significantly higher in the LRAF group than no-LRAF group $(\mathrm{p}=0.021, \mathrm{p}=0.011)$. The $\Delta \mathrm{NLR}$ in the initial ablation sessions was significantly higher in patients with right $\mathrm{PVs}$ reconnections than in those without in the repeat ablation sessions $(0.914 \pm 1.199$ vs $2.316 \pm 3.615, \mathrm{p}=0.032)$. Conclusion The NLR and PLR changes were associated with an LRAF. A higher NLR change in the initial ablation session was associated with a right $\mathrm{PV}$ reconnection in the repeat ablation session.
\end{abstract}

Inflammation markers, neutrophil-to-lymphocyte ratio and platelet-to lymphocyte ratio after pulmonary vein isolation, were associated with late clinical outcomes and pulmonary vein reconnections

Masamichi Yano, MD, PhD; Yasuyuki Egami, MD; Kohei Ukita, MD; Akito Kawamura, MD; Hitoshi Nakamura, MD; Yutaka Matsuhiro, MD; Koji Yasumoto, MD;

Masaki Tsuda, MD; Naotaka Okamoto, MD; Akihiro Tanaka, MD;

Yasuharu Matsunaga-Lee, MD; Ryu Shutta, MD; Masami Nishino, MD, PhD, FACC, FESC; Jun Tanouchi $\mathrm{MD}, \mathrm{PhD}$

Division of Cardiology, Osaka Rosai Hospital,

3-1179 Nagasonecho, kita-ku, Sakai, Osaka, 591-8025, Japan

\section{Correspondence to:}


Masami Nishino, MD, PhD, FACC, FESC

Division of Cardiology, Osaka Rosai Hospital, Sakai, Osaka, Japan

3-1179 Nagasonecho, kita-ku, Sakai, Osaka, 591-8025, Japan

TEL: +81-72-252-3561

FAX: +81-72-255-3349

E-mail:mnishino@osakah.johas.go.jp

\section{Abstract}

\section{Background}

Previous reports demonstrated that the early recurrence of atrial fibrillation (ERAF) after pulmonary vein isolation (PVI) was associated with the inflammatory process. The neutrophil to lymphocyte ratio (NLR) and platelet to lymphocyte ratio (PLR) have been proposed as indicators of a systemic inflammatory response. However the relationship between NLR/PLR and clinical outcomes after PVI remains unclear.

Methods and Results We enrolled 633 consecutive atrial fibrillation (AF) patients who underwent PVI from September 2014 to June 2018. The NLR and PLR in peripheral blood leukocytes 1 day before and 36-48 hours after PVI were measured. We compared the NLR and PLR changes in each parameter $(\Delta$ NLR and $\triangle$ PLR) between a late recurrence of AF group after the PVI (LRAF, during 1 year after PVI) and the no-LRAF group. We also evaluated the relationship between the $\Delta$ NLR / $\triangle \mathrm{PLR}$ and pulmonary vein (PV) reconnections in repeated ablation procedures. The LRAF patients consisted of 151 patients $(23.9 \%)$. The $\triangle \mathrm{NLR}$ and $\triangle \mathrm{PLR}$ were significantly higher in the LRAF group than no-LRAF group $(\mathrm{p}=0.021, \mathrm{p}=0.011)$. The $\triangle$ NLR in the initial ablation sessions was significantly higher in patients with right PVs reconnections than in those without in the repeat ablation sessions ( $0.914 \pm 1.199$ vs $2.316 \pm 3.615, \mathrm{p}=0.032$ ).

\section{Conclusion}

The NLR and PLR changes were associated with an LRAF. A higher NLR change in the initial ablation session was associated with a right PV reconnection in the repeat ablation session.

Key Words: atrial fibrillation; pulmonary vein isolation; inflammation; recurrence of atrial fibrillation after pulmonary vein isolation; neutrophil-to-lymphocyte ratio; platelet-to-lymphocyte ratio; left atriumpulmonary vein reconnection.

\section{INTRODUCTION}

Atrial fibrillation (AF) is the most common arrhythmia. Pulmonary vein isolation (PVI) has become established as a standard therapy for patients with drug-refractory paroxysmal atrial fibrillation $(\mathrm{PAF})^{1-3}$ since extra-systoles from pulmonary veins (PV) are the most common AF trigger activity. ${ }^{4}$ The efficacy and safety of the PVI of AF have been reported in several randomized trials. ${ }^{5-7}$ There are many studies that have evaluated the predictors of recurrences after PVI. ${ }^{8-10}$ The inflammatory response level after ablation was significantly associated with early recurrence of AF/atrial tachycardia (AT) (ERAF) within 3 days after the procedure. ${ }^{11-12}$ Several reports have shown that the incidence of an ERAF after PVI is approximately $40 \%$ and ERAFs are only a transient phenomenon attributed to the ablation related inflammatory process. ${ }^{13}$ On the contrary, there was a report that showed AF recurrence within 48 hours of a PVI was a predictor of a poor clinical outcome on follow-up. ${ }^{14}$ The relationship between the inflammation level and hemogram parameters such as the neutrophil-to-lymphocyte ratio (NLR) and platelet-to-lymphocyte ratio (PLR) have been proposed as reliable indicators of the immune activation and inflammation. ${ }^{15-16}$ Their measurements have proven to be useful prognostic biomarkers in cardiovascular disease. ${ }^{17}$ However, it is unclear whether the NLR and PLR are associated with ERAFs or late recurrences of AF (LRAFs). Therefore, in the present study, we aimed to investigate the relationship between the hemogram parameters (NLR and PLR) and 
ERAFs and LRAFs after the PVI. Secondly, we aimed to investigate the association between the hemogram parameters and electrophysiological findings in the repeat ablation sessions.

\section{METHODS}

\subsection{Study populations}

We enrolled AF patients who underwent a PVI from September 2014 to June 2018 in our hospital. All patients received a detailed informed consent and the study protocol was approved by the hospital's institutional review board.

\subsection{Echocardiography study}

All patients underwent transthoracic echocardiography before the ablation. The left atrial diameter was measured at end-systole on the M-mode image obtained from the parasternal long-axis view. Transthoracic echocardiography was performed with a $5 \mathrm{MHz}$ multiplane probe and live images were interpreted by experienced physicians who were blinded to the outcome of the ablation. Transesophageal echocardiography prior to the PVI was performed to exclude any left atrial or left atrial appendage thrombi.

\subsection{Ablation procedure}

All antiarrhythmic drugs were discontinued for at least 5 half-lives before the ablation. In our hospital, all AADs were discontinued before PVI because the previous study demonstrated AADs, in particular $\mathrm{Na}^{+}$ channel blocker, suppressed extra systole from pulmonary vein. ${ }^{18}$ Anticoagulation therapy was started at least 3 weeks before the ablation procedure. A bolus infusion of $25 \mathrm{mg}$ of hydroxyzine pamoate and $15 \mathrm{mg}$ of pentazocine were intravenously administered before the ablation procedure. The ablation procedure was performed under mild sedation obtained with propofol and dexmedetomidine and the patients received adaptive servoventilation. An esophagus temperature monitoring catheter via the nose was placed. A duo-decapolar catheter (BeeAT, Japan Lifeline Co., Tokyo, Japan) was placed in the coronary sinus through the right internal jugular vein. If the patient was in AF, internal atrial cardioversion was performed with 15-20J of biphasic energy. We performed a transseptal puncture under guidance with the SoundStar 3D Ultrasound Catheter (Biosense Webster, Diamond Bar, CA, USA) from the right atrium. After the transseptal puncture, 2 long sheaths (8.5Fr SL0, Abbott, Chicago, IL, USA) were inserted into the left atrium. A $100 \mathrm{IU} / \mathrm{kg}$ body weight bolus of heparin was administered following the transseptal puncture and heparinized saline was continuously infused to maintain the activated clotting time at 300-350 second. One circular mapping catheter was deployed in the superior and inferior PVs and the left-sided then right-sided ipsilateral PVs were circumferentially ablated guided by three-dimensional left atrium mapping (CARTO3, Biosense-Webster, Diamond Bar, CA, USA). The PVI was performed with a $3.5 \mathrm{~mm}$ ablation catheter with an externally-irrigated tip (ThermoCool@ SmartTouch@ Catheter, Biosense-Webster, Diamond Bar, CA, USA). Radiofrequency current was delivered with a power of up to $30 \mathrm{~W}$ and limited to $20 \mathrm{~W}$ near the esophagus for 25 seconds. The PVI was considered successful when all ostial PV potentials were abolished during coronary sinus pacing and was also confirmed by PV pacing under an isoproterenol (under increasing heart rate) and ATP bolus administration (40mg). Cavo-tricuspid isthmus block was performed when isthmus dependent atrial flutter was clinically confirmed or under the operators' judgement. When AF persisted after the PVI or firing sites of atrial premature contraction triggers were detected, a substrate modification was sequentially performed.

\subsection{Biomarkers}

We measured the white blood cells (WBCs), hemograms (neutrophils, lymphocytes and monocytes), platelets, and high sensitive C-reactive protein (hs-CRP) levels 1 day before and 36-48 hours after the ablation. We calculated the NLR and PLR before and after the ablation, respectively. The $\triangle$ NLR and $\triangle \mathrm{PLR}$ were calculated as below:

$\Delta \mathrm{NLR}=[\mathrm{NLR}$ after ablation $]-[$ NLR before ablation $], \Delta \mathrm{NLR}=[$ PLR after ablation $]-[$ PLR before ablation], respectively.

\subsection{Follow-up}


After the ablation, no antiarrhythmic medications were prescribed. The patients underwent continuous electrocardiogram (ECG) monitoring for approximately 3 days (until discharge) after the ablation. They came to our cardiology clinic 1 month after the ablation. Subsequent follow-ups were performed every 3 months at the clinic. The follow-up visits included a clinical interview, ECG, blood examination, 24 hour Holter monitoring or portable ECG (2 week cardiac event recording), and echocardiography. Patients with palpitations or other chest symptoms underwent a portable ECG. Recurrence after the ablation was defined as $\mathrm{AF} / \mathrm{AT}$ documented on the ECG or AF/AT continuing longer than 30 seconds on the Holter or portable ECG. AF/AT during the first 3 months after the ablation (blanking period) was considered as an ERAF and AF/AT of more than 3 months after the ablation was considered as an LRAF.

\subsection{Statistical analysis}

JMP 14 statistical software (SAS Institute Inc., Cary, North Carolina, USA) was used for the statistical analysis. Continuous parameters were expressed as the mean \pm standard deviation. Two-group comparisons were analyzed by an unpaired 2-tailed Student's $t$ test. Categorical data were expressed as the number (percentage) and were compared using the Chi-square test. A multiple-group comparison was performed by a one-way ANOVA followed by the Tukey-Kramer HSD test for a comparison of the means.

\section{RESULTS}

\subsection{Patient characteristics}

We enrolled 633 AF patients who underwent a PVI from September 2014 to June 2018 in our hospital. The clinical characteristics of the patients in the LRAF and non-LRAF groups are shown in Table 1. The age, body surface area, ratio of hypertension, and diabetes mellitus did not differ significantly between the two groups. The ratio of females, chronic heart failure, and a history of a stroke were higher in the LRAF group than non-LRAF group ( $\mathrm{p}=0.024, \mathrm{p}=0.040, \mathrm{p}=0.037$, respectively). There was a significant difference in the CHADS2 VASC score between the two groups $(\mathrm{p}=0.023)$ due to the ratio of females. The ratio of PAF was higher in the LRAF group than non-LRAF group $(\mathrm{p}<0.001)$. Plasma brain natriuretic peptide (BNP) level was significantly higher in LRAF group than in non-LRAF group $(195.2 \pm 217.5 \mathrm{pg} / \mathrm{ml}$ vs $144.7 \pm 169.3 \mathrm{pg} / \mathrm{ml}, \mathrm{p}=0.003)$. In the echocardiographic parameter, left ventricular end-diastolic diameter (LVDd), left ventricular end-systolic diameter (LVDs), and left ventricular ejection fraction (LVEF) did not differ significantly between the two groups. The mean left atrium diameter (LAD) was $45.8 \pm 5.27 \mathrm{~mm}$ and $44.1 \pm 6.16 \mathrm{~mm}$ in the LRAF and non-LRAF groups, respectively $(\mathrm{p}=0.003)$. The ratio of severe mitral regurgitation (MR) and tricuspid regurgitation (TR) did not differ between the two groups.

\subsection{Oral medication}

The oral medications before the ablation are shown in Table 1. The ratio of the patients administered direct oral anticoagulants was significantly higher in the non-LRAF group than LRAF group. The ratio of the patients administered anti-arrhythmic drugs, beta-blockers, angiotensin-converting enzyme inhibitors/angiotensin II receptor blockers, digitalis, and statins did not differ significantly between the two groups.

\subsection{Procedure characteristics}

The procedural characteristics of the patients in the LRAF and non-LRAF groups are shown in Table 2. The ratio of cavo-tricuspid isthmus block, superior vena cava isolation, and left atrium posterior wall isolation did not differ significantly between the two groups. The ratio of a non-PV trigger ablation and atrial tachycardia ablation was significantly higher in the LRAF group than non-LRAF group $(\mathrm{p}<0.001, \mathrm{p}=0.021$, respectively)

\subsection{Biomarkers associated with inflammation}

The average follow-up duration was $2.7 \pm 1.3$ years. There were no significant differences in the number of the WBCs, ratio of the neutrophils, and lymphocytes before the ablation between the LRAF and non-LRAF groups (Table 3). No significant difference was demonstrated in the CRP level and number of platelets before the ablation between the two groups (Table 3). After ablation there were also no significant differences in these biomarkers between the two groups (Table 3). 
The ERAF patients consisted of 203 patients $(32.1 \%)$. The $\triangle$ NLR and $\triangle$ PLR were significantly higher in the ERAF than no-ERAF group $(1.464 \pm 2.756$ vs $0.779 \pm 1.464,6.390 \pm 60.76$ vs $-2.751 \pm 41.38, \mathrm{p}<0.001$, $\mathrm{p}=0.027$, respectively).

Between the LRAF and no-LRAF groups, the NLR after ablation and $\triangle$ NLR were significantly higher in the LRAF and no-LRAF groups $(3.747 \pm 3.033$ vs $3.288 \pm 1.766,1.324 \pm 2.911$ vs $0.896 \pm 1.596, \mathrm{p}=0.022$, $\mathrm{p}=0.021$, respectively), while there were no significant differences in the NLR before the ablation $(2.422 \pm$ 1.480 vs $2.392 \pm 1.173, \mathrm{p}=0.793$, Figure $1 \mathrm{~A})$. The PLR after the ablation and $\Delta \mathrm{PLR}$ were significantly higher in late recurrence and no-late recurrence groups (147.0 \pm 80.09 vs $133.4 \pm 58.87,8.993 \pm 61.61$ vs -2.569 $\pm 43.43, \mathrm{p}=0.022, \mathrm{p}=0.011$, respectively), but there were no significant differences in the PLR before the ablation between the 2 groups (135.8 \pm 59.65 vs $136.0 \pm 53.90, \mathrm{p}=0.981$, Figure1B).

\subsection{Electrophysiological findings in repeat ablation sessions}

The LRAF group consisted of 151 patients (23.9\%) and repeat ablation sessions were performed in 76 patients in the LRAF group $(50.3 \%)$. The average duration from the 1st session to the repeat ablation session was $8.6 \pm 7.1$ months. A reconnection of any of the PVs was identified in 55 of 76 patients (72.4\%). No significant difference was observed in the $\triangle$ NLR between the patients without a PV reconnection and those with a PV reconnection $(1.120 \pm 1.323$ vs $1.880 \pm 3.234, \mathrm{p}=0.302$, Figure $2 \mathrm{~A})$. However, the $\Delta$ NLR was significantly higher in the patients with a right $\mathrm{PV}$ reconnection than in those without a right $\mathrm{PV}$ reconnection $(0.914 \pm 1.199 \mathrm{vs}$ $2.316 \pm 3.615, \mathrm{p}=0.032$, Figure $2 \mathrm{~B})$. No significant difference was observed in the $\Delta$ PLR between the patients without a PV reconnection and those with a PV reconnection ( $8.233 \pm 45.00$ vs $21.84 \pm 74.11, \mathrm{p}=0.434$, Figure $2 \mathrm{C})$. No significant difference was observed in the $\triangle \mathrm{PLR}$ between the patients with a right PV reconnection and those without a right PV reconnection (10.20 36.54 vs $24.82 \pm 85.21, \mathrm{p}=0.349$, Figure 2D).

In 55 patients, $1 \mathrm{PV}, 2 \mathrm{PV}, 3 \mathrm{PV}$, and $4 \mathrm{PV}$ reconnections in repeat ablation sessions were detected in $12,23,7$, and 13 patients, respectively. There was a tendency for the $\triangle$ NLR to increase as the number of reconnected PVs increased, but there was no significant difference in the $\triangle$ NLR between the two groups (Figure 3 ).

\section{DISCUSSION}

The present study highlighted the following results. (1) Higher NLR and PLR changes were associated with a late recurrence of AF/AT after the PVI. (2) An NLR change was significantly higher in the patients with right $\mathrm{PV}$ reconnections than in those without right $\mathrm{PV}$ reconnections.

Radiofrequency catheter ablation of atrial arrhythmias is known to cause an increase in various markers of inflammation and myocardial injury. ${ }^{19-20}$ Infiltration of inflammatory cytokines (IL-1, IL-8, TNF- $\alpha$ ) into myocardial injury sites induces hematopoietic factors. ${ }^{21-22}$ These factors stimulate hematopoiesis of neutrophils in bone marrow and a rapid supply of neutrophils from the bone marrow pool. Although segmented cells are the main neutrophils in peripheral blood, in an inflammatory status, the number of stab cells and metamyelocytes increases. Increasing the number of neutrophils also leads to an enhancement in the blood viscosity and hypercoagulability by causing an interaction in the platelets and endothelium. ${ }^{23}$ Neutrophils suggest nonspecific inflammation and the decrease in the number of lymphocytes reflects the inflammation. ${ }^{24}$ Low lymphocyte counts have been reported to be related to inflammation. ${ }^{25}$ The NLR, therefore, more effectively reveals inflammation than the neutrophil count. ${ }^{23}$ The NLR is a powerful independent indicator of a prognosis in systemic inflammation, atherosclerotic heart disease, and cancer patients. ${ }^{26-27}$ The PLR is an easy to perform blood test associated with a poor prognosis when elevated in patients suffering from various oncologic disorders. ${ }^{28-29}$ The PLR is also a strong independent indicator of a prognosis in systemic inflammation such as in critical limb ischemia and collagen disease. ${ }^{30-31}$

A previous study demonstrated that inflammation was increasingly recognized to play a significant role in the genesis and perpetuation of AF. ${ }^{32-33}$ Koyama et al. reported an immediate AF recurrence is closely associated with an acute inflammatory process and over $70 \%$ of patients with an early AF recurrence have AF episodes 3 months after ablation. ${ }^{11}$ The relationship between an ERAF and LRAF remains unclear, but several reports have shown the relationship between an ERAF and the durability of the $\mathrm{PVI}^{34}$, and 
overt ERAFs require the reconnection of a critical number of fibers at the level of the PV-LA junction. ${ }^{35}$ Probably the reconnection of the left atrium and PVs, presence of non-PV foci triggering AF, and other mechanisms might be associated with LRAFs, but according to the relationship between an ERAF and LRAF, an acute inflammatory response after a PVI is one of the most important risk factors for an LRAF. In our study, the $\triangle$ NLR and $\triangle \mathrm{PLR}$ were higher in patients with an ERAF than in the no-ERAF group, and as a result, the mechanism of the ERAF was possibly associated with an inflammatory process. We also demonstrated that the $\triangle \mathrm{NLR}$ was higher in patients with a right PV reconnection than in those without a right $\mathrm{PV}$ reconnection $(0.914 \pm 1.199$ vs $2.316 \pm 3.615, \mathrm{p}=0.032$, Figure $2 \mathrm{~B})$. Recent reports have shown that patients with an ERAF have a significantly higher rate of PV reconnections, in particular of right PV reconnections $(82.5 \%$ vs $29.2 \%) .{ }^{35}$ They also showed that a higher number of right PVI segments with reconnections were observed in those with an ERAF as compared to those without. It is unclear why the right PV reconnections were associated with ERAFs. Presently it remains unclear whether a durable right PV isolation will positively impact the ERAF expression and long-term outcomes. A hypothesis to explain this result is that radiofrequency current is delivered with a high power on the right PV anterior line, which has a thick wall and the posterior line consisting of fiber bundles, while the radiofrequency current is limited on the left posterior line near the esophagus.

In the LRAF group, the ratio of persistent AF (PerAF) patients was significantly higher than that in the noLRAF group. The plasma BNP level was also significantly higher in the LRAF group than no-LRAF group. Generally the left atrium in PerAF patients causes structural and electrical remodeling and a progressive pressure overload. The level of inflammatory cytokines in heart failure patients was significantly higher than that in patients with a normal heart, but in this study there were no significant differences in the WBCs, hs-CRP, or NLR levels before the ablation between the PAF and PerAF patients. These levels after the ablation and changes from before to after the ablation were similar between the PAF and PerAF patients. These results indicated that the difference between PAF and PerAF did not affect the inflammatory status during the acute phase of the ablation. In the LRAF group, the ratio of a non-PV trigger ablation and an AT ablation was higher than that in the no-LRAF group due to more PerAF patients in the recurrence group, but the total number of energy applications did not differ the between the two groups. A variation was observed in the period from the initial to repeat ablation session (8.6 \pm 7.1 months), but this also did not differ between the two groups.

The NLR and PLR can be easily calculated by dividing the neutrophil and platelet counts by the number of lymphocytes, respectively. These measurements are a simple, cost-effective routine test. As suggested by many studies, they may emerge as markers of inflammatory conditions and disease activity. In the present study we demonstrated the relationship between the ERAF/LRAF and $\triangle \mathrm{NLR} / \Delta \mathrm{PLR}$, suggesting an inflammation status. Further studies on inflammation after the PVI and AF recurrence and PV reconnections may be necessary in the future.

\section{CONCLUSION}

The NLR and PLR changes were significantly related to early and late recurrences of AF/AT after the PVI. A greater NLR change was significantly associated with a right PV reconnection in the repeat ablation session.

\section{Acknowledements}

The authors thank Mr. John Martin for his linguistic assistance with this manuscript.

\section{Disclosure}

Nothing to disclose

\section{References}

1. Haïssaguerre M, Shah DC, Jaïs P et al. Electrophysiological breakthroughs from the left atrium to the pulmonary veins. Circulation 2000;102:2463-2465. 
2. Nault I, Miyazaki S, Forclaz A et al. Drugs vs. ablation for the treatment of atrial fibrillation: the evidence supporting catheter ablation. Eur Heart J. 2010;31:1046-1054.

3. Calkins H, Kuck KH, Cappato R et al. 2012 HRS/EHRA/ECAS expert consensus statement on catheter and surgical ablation of atrial fibrillation: recommendations for patient selection, procedural techniques, patient management and follow-up, definitions, endpoints, and research trial design: a report of the Heart Rhythm Society (HRS) Task Force on Catheter and Surgical Ablation of Atrial Fibrillation. Heart Rhythm. 2012;9:632-696.

4. Haïssaguerre M, Jaïs P, Shah DC et al. Spontaneous initiation of atrial fibrillation by ectopic beats originating in the pulmonary veins. N Engl J Med. 1998;339:659-666.

5. Parkash R, Tang AS, Sapp JL, Wells G. Approach to the catheter ablation technique of paroxysmal and persistent atrial fibrillation: a meta-analysis of the randomized controlled trials. J Cardiovasc Electrophysiol. 2011;22:729-738.

6. Pappone C, Augello G, Sala S et al. A randomized trial of circumferential pulmonary vein ablation versus antiarrhythmic drug therapy in paroxysmal atrial fibrillation: the APAF Study. J Am Coll Cardiol. 2006;48:2340-2347.

7. Calkins H, Reynolds MR, Spector P et al. Treatment of atrial fibrillation with antiarrhythmic drugs or radiofrequency ablation: two systematic literature reviews and meta-analyses. Circ Arrhythm Electrophysiol. 2009;2:349-361.

8. Tang RB, Dong JZ, Liu XP et al. Obstructive sleep apnoea risk profile and the risk of recurrence of atrial fibrillation after catheter ablation. Europace. 2009;11:100-105.

9. D'Ascenzo F, Corleto A, Biondi-Zoccai G et al. Which are the most reliable predictors of recurrence of atrial fibrillation after transcatheter ablation?: a meta-analysis. Int J Cardiol. 2013;167:1984-1989.

10. Zhuang J, Wang Y, Tang $\mathrm{K}$ et al. Association between left atrial size and atrial fibrillation recurrence after single circumferential pulmonary vein isolation: a systematic review and meta-analysis of observational studies. Europace. 2012;14:638-645.

11. Koyama T, Sekiguchi Y, Tada H et al. Comparison of characteristics and significance of immediate versus early versus no recurrence of atrial fibrillation after catheter ablation. Am J Cardiol. 2009;103:124954.

12. Lim HS, Schultz C, Dang J et al. Time course of inflammation, myocardial injury, and prothrombotic response after radiofrequency catheter ablation for atrial fibrillation. Circ Arrhythm Electrophysiol. 2014;7:83-89.

13. Andrade JG, Khairy P, Verma A et al. Early recurrence of atrial tachyarrhythmias following radiofrequency catheter ablation of atrial fibrillation. Pacing Clin Electrophysiol. 2012;35:106-116.

14. Richter B, Gwechenberger M, Socas A, Marx M, Gössinger HD. Frequency of recurrence of atrial fibrillation within 48 hours after ablation and its impact on long-term outcome. Am J Cardiol. 2008;101:843847.

15. Ijsselmuiden AJ, Musters RJ, de Ruiter G et al. Circulating white blood cells and platelets amplify oxidative stress in heart failure. Nat Clin Pract Cardiovasc Med. 2008;5:811-820.

16. Panni RZ, Lopez-Aguiar AG, Liu J et al. Association of preoperative monocyte-to-lymphocyte and neutrophil-to-lymphocyte ratio with recurrence-free and overall survival after resection of pancreatic neuroendocrine tumors (US-NETSG). J Surg Oncol. 2019;120:632-638.

17. Afari ME, Bhat T. Neutrophil to lymphocyte ratio (NLR) and cardiovascular diseases: an update. Expert Rev Cardiovasc Ther. 2016;14:573-577.

18. Kumagai K, Tojo H, Noguchi H et al. Effects of the NA+ channel blocker pilsicainide on the electrophysiologic properties of pulmonary veins in patients with atrial fibrillation. J Cardiovasc Electrophysiol. 2004;15:1396-1401.

19. Lellouche N, Sacher F, Wright M Usefulness of C-reactive protein in predicting early and late recurrences after atrial fibrillation ablation. Europace. 2009;11:662-664.

20. Katritsis D, Hossein-Nia M, Anastasakis A et al. Use of troponin-T concentration and kinase isoforms for quantitation of myocardial injury induced by radiofrequency catheter ablation. Eur Heart J. 1997;18:1007-1013. 
21. Summers C, Rankin SM, Condliffe AM, Singh N, Peters AM, Chilvers ER. Neutrophil kinetics in health and disease. Trends Immunol. 2010;31:318-324.

22. Zhan Y, Lieschke GJ, Grail D, Dunn AR, Cheers C. Essential roles for granulocyte-macrophage colony-stimulating factor (GM-CSF) and G-CSF in the sustained hematopoietic response of Listeria monocytogenes-infected mice. Blood. 1998;91:863-869.

23. Li X, Ji Y, Kang J et al. Association between blood neutrophil-to-lymphocyte ratio and severity of coronary artery disease: Evidence from 17 observational studies involving 7017 cases. Medicine (Baltimore). 2018;97:e12432.

24. Diakos CI, Charles KA, McMillan DC, Clarke SJ. Cancer-related inflammation and treatment effectiveness. Lancet Oncol. 2014;15:e493-503.

25. Gravani F, Papadaki I, Antypa E et al. Subclinical atherosclerosis and impaired bone health in patients with primary Sjogren's syndrome: prevalence, clinical and laboratory associations. Arthritis Res Ther. $2015 ; 17: 99$.

26. Lin J, Zhang W, Huang Y et al. Sarcopenia is associated with the neutrophil/lymphocyte and platelet/lymphocyte ratios in operable gastric cancer patients: a prospective study. Cancer Manag Res. 2018;10:4935-4944.

27. Nam KW, Kwon HM, Jeong HY, Park JH, Kim SH, Jeong SM. High neutrophil to lymphocyte ratios predict intracranial atherosclerosis in a healthy population. Atherosclerosis. 2018;269:117-121.

28. Suades R, Padro T, Vilahur G et al. Circulating and platelet-derived microparticles in human blood enhance thrombosis on atherosclerotic plaques. Thromb Haemost 2012;108:1208-1219.

29. Kwon HC, Kim SH, Oh SY et al. Clinical significance of preoperative neutrophil-lymphocyte versus platelet-lymphocyte ratio in patients with operable colorectal cancer. Biomarkers 2012;17:216-222.

30. Gary T, Pichler M, Belaj K et al. Platelet-to-lymphocyte ratio: a novel marker for critical limb ischemia in peripheral arterial occlusive disease patients. PLoS One. 2013;8:e67688

31. Qin B, Ma N, Tang Q et al. Neutrophil to lymphocyte ratio (NLR) and platelet to lymphocyte ratio (PLR) were useful markers in assessment of inflammatory response and disease activity in SLE patients. Mod Rheumatol. 2016;26:372-376.

32. Issac TT, Dokainish H, Lakkis NM. Role of inflammation in initiation and perpetuation of atrial fibrillation: a systematic review of the published data. J Am Coll Cardiol. 2007;50:2021-2028.

33. Aviles RJ, Martin DO, Apperson-Hansen C et al. Inflammation as a risk factor for atrial fibrillation. Circulation. 2003;108:3006-10.

34. Das M, Wynn GJ, Morgan M et al. Recurrence of atrial tachyarrhythmia during the second month of the blanking period is associated with more extensive pulmonary vein reconnection at repeat electrophysiology study. Circ Arrhythm Electrophysiol. 2015;8:846-52.

35. Mujović N, Marinković M, Marković N et al. The relationship of early recurrence of atrial fibrillation and the 3-month integrity of the ablation lesion set. Sci Rep. 2018;8:9875.

\section{Figure legends}

Figure 1

Hemogram parameters (NLR, MLR and PLR) and the change in the NLR between the late recurrence group and no-late recurrence group. (A) The NLR before and after the ablation and the change in the NLR $(\triangle \mathrm{NLR})$. (B) The PLR before and after the ablation and the change in the PLR ( $\triangle \mathrm{PLR})$.

Figure 2

The relationship between the $\triangle \mathrm{NLR}$ in the initial ablation session and LA-PV reconnections in the repeat ablation session

(A) The $\triangle$ NLR between the patients with and without PV reconnections

(B) The $\triangle$ NLR between the patients with and without right PV reconnections

(C) The $\triangle$ PLR between the patients with and without PV reconnections 
(D) The $\triangle \mathrm{PLR}$ between the patients with and without right PV reconnections

Figure 3

The relationship between the $\triangle$ NLR in the initial ablation session and number of reconnected PVs in the repeat ablation session

(A)

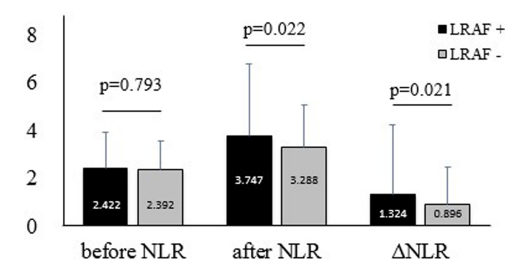

(A)

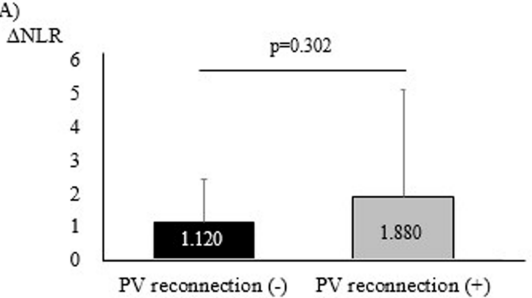

(C)

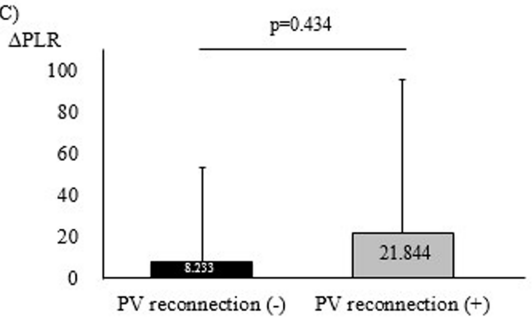

(B)

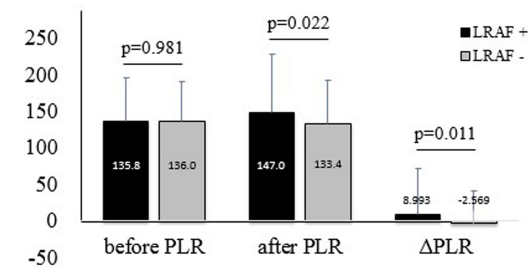

(B)

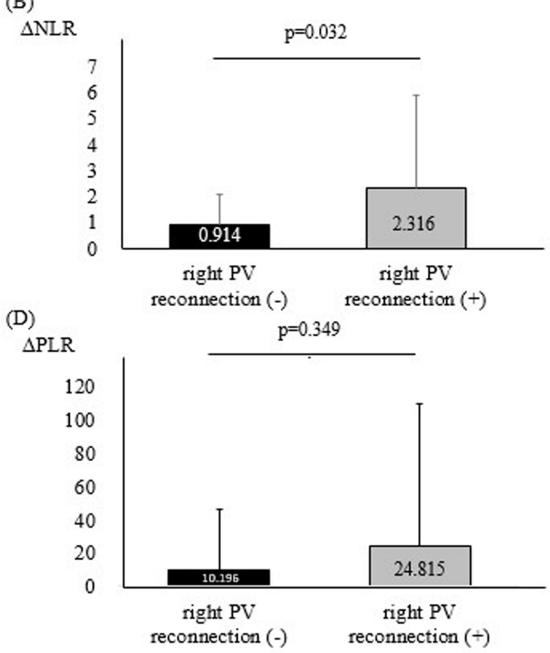

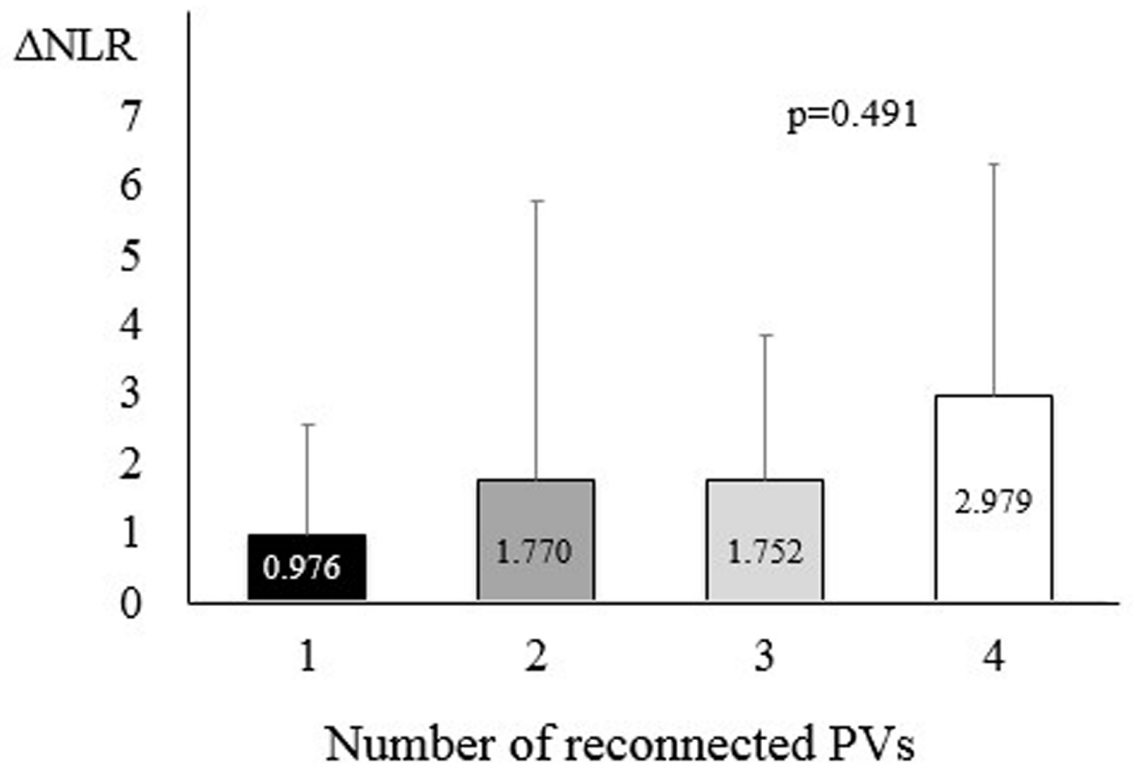

